\title{
Efficiency Enhancement in a Multi-coil Wireless Power Transfer System
}

\author{
Samuel Afoakwa, Faculty of Engineering, Ghana Communication Technology University, Tesano, Accra, Ghana \\ samafoak@gmail.com
}

Kyei Anim, Department of Electronic Engineering, Hanbat National University, Yuseong-gu, Daejeon, South Korea kyeianim@gmail.com

Young-Bae Jung, Department of Electronic Engineering, Hanbat National University, Yuseong-gu, Daejeon, South Korea ybjung@hanbat.ac.kr

\begin{abstract}
Wireless power transfer technology via magnetic resonance coupling now has significant interest in industry and research with many applications. This paper proposes a linear multiple transmitter coil array (5 coils) for wireless power transfer for added gain and hence higher transfer efficiency in comparison to a single transmitter coil. The frequency splitting effect as a result of the coupling between the resonant transmitter coils due to their close proximity is shown to reduce the transfer efficiency to a receiver. The effect of the array spacing on splitting effect suppression is verified. It is shown that the splitting effect is sup-pressed as the distance between the coils is increased leading to a higher received signal and hence higher efficiency. Proposed horizontal displacement of the middle transmitter coils (2nd and 4th coils) in the coil array is shown to suppress frequency splitting. To further suppress the splitting effect due to the magnetic coupling between the transmitter coils, a multiple transmitter array is proposed with different coil turns. Thus it is shown that designing the multiple coil array with mixed number of coil turns (the 2nd and 4th coils are designed to have different number of turns as compared to the other three coils) causes uniform coupling among the coils reducing and eventually eliminating the splitting effect. Also to increase the efficiency at the receiver coil, displaced stacked coils are introduced on top of the coil array. The pro-posed stacked coil array is demonstrated to improve the transfer efficiency. Using the techniques, the proposed linear array structure achieves a transfer efficiency of $36.9 \%$ for a receiver coil at the boresight of the array at a transfer distance of $40 \mathrm{~cm}$.
\end{abstract}

Keywords:

Multiple transmitter array, resonant coupling, frequency splitting

Article Received: 18 October 2020, Revised: 3 November 2020, Accepted: 24 December 2020

\section{INTRODUCTION}

Wireless power transfer is electrical energy transmission using time varying electromagnetic fields without the use of wires. Thus wireless power transfer provides a useful solution of powering electrical devices in situations where interconnecting wires are a downside, hazardous or not applicable.

High efficiency is achieved with inductive coupling as far as the coils are close together and on the same axis. Thus to increase the efficiency at larger distances, most modern inductive systems use resonant inductive coupling [1-4].

Different transmitter and receiver configurations are developed depending on the application.

The use of multiple transmitters is shown in [5] to enhance the gain and also have diversity effects.
The increase in the number of transmitters was found to increase the gain of the system, hence increase in the transfer efficiency. This phenomenon is shown to occur when the transmitters are far away from each other such that there is negligible coupling between adjacent coils. There is transmitter resonant coupling between two transmitters as the transmitters are brought closer to each other. This coupling causes a splitting effect and therefore a reduction of the gain. A solution for suppressing and eventually eliminating the splitting effect is however not provided.

In this paper a linear multiple transmitter coil structure is proposed with methods of enhancing the transfer efficiency of the array to a single receiver at a transfer distance $40 \mathrm{~cm}$. Due to the 
coupling between adjacent transmitter coils, there is a frequency splitting effect of the received signal at the receiver. This phenomenon reduces the transfer efficiency at the receiver and hence suppression and elimination of the splitting effect increases the efficiency [6, 7]. The design of a proposed structure where the middle coils in the multiple coil array are displaced horizontally suppresses and eventually eliminates the splitting effect and hence enhances the efficiency. The splitting effect can also be suppressed by the design of a mixed number of turns coil array where the middle coils are designed with different number of turns causing uniform coupling between adjacent coils. To enhance the transfer efficiency further, stacked coils are introduced above the coil array. As compared to the multiple transmitter coil structure in [6] which achieves a transfer efficiency of $11 \%$ at $40 \mathrm{~cm}$, the proposed structure achieves $36.9 \%$.

\section{MULTIPLE-TRANSMITTER COIL DESIGN OVERVIEW}

\section{A) Equal Turn-Number Transmitter Array}

The design of the multiple transmitter array is initially designed by using the same number of coil turns. The number of turns of the resonator coil increases the quality factor $\mathrm{Q}$ of the coil. The higher the number of turns the higher the quality factor of the resonator. First each of the transmitter coils are designed with an inner radius of $8.37 \mathrm{~cm}$, with 17 turns having a turns spacing of $1.46 \mathrm{~mm}$ with a $2.54 \mathrm{~mm}$ copper wire. The coil array spacing, $s$ of the array made of five resonators is set at $35 \mathrm{~cm}$ from the centers of adjacent coils. The receiver coil which has the same parameters as the transmitter coils is placed at $40 \mathrm{~cm}$ away (transfer distance $D$ ) in the design. The transmitter array design structure is shown in Fig. 1a and $1 \mathrm{~b}$ with the structure indicating the transfer distance $D$ is illustrated in Fig. 1c.

Secondly, an array is designed with each coil made of a start radius of $9.5 \mathrm{~cm}$ with turns spacing of $1.46 \mathrm{~mm}$ with a $2.54 \mathrm{~mm}$ copper wire just as with the first array. However, the number of turns of the coil is 15 . The structure of the single coil used for this transmitter array is shown in Fig. 1d. The transmitter array and the single receiver coil is simulated as a two port network with the transmitters combined into a single port and the receiver coil as the other port as is illustrated in Fig. 2.

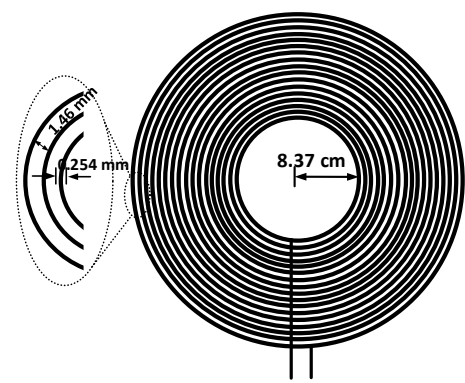

(a)

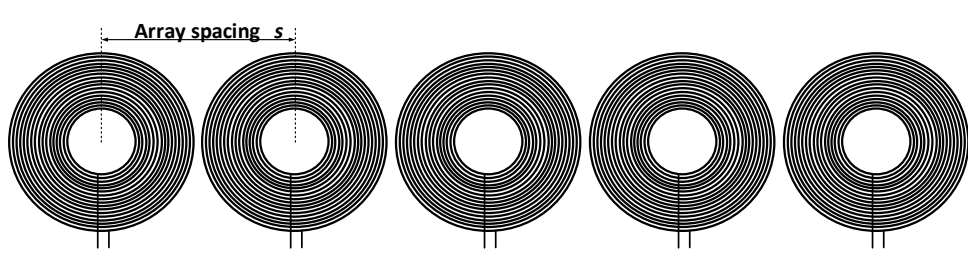

(b)

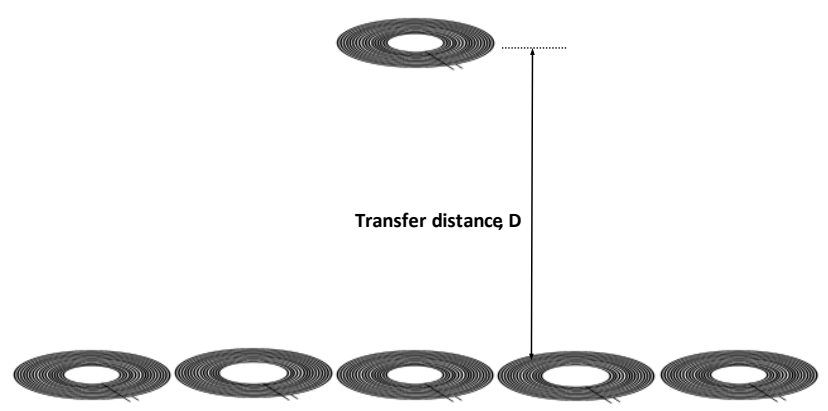

(c)

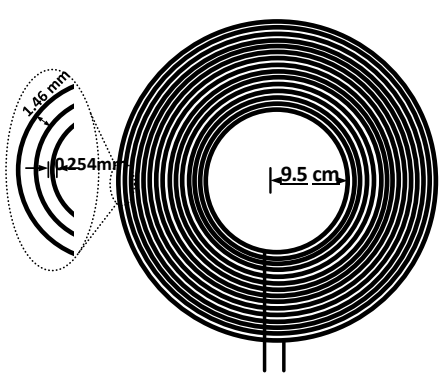

(d)

Figure 1. Multiple transmitter coil structure (a) 17-turn coil (b) Coil array structure showing array spacing s (c) Coil array structure showing the transfer distance (d) 15-turn coil 


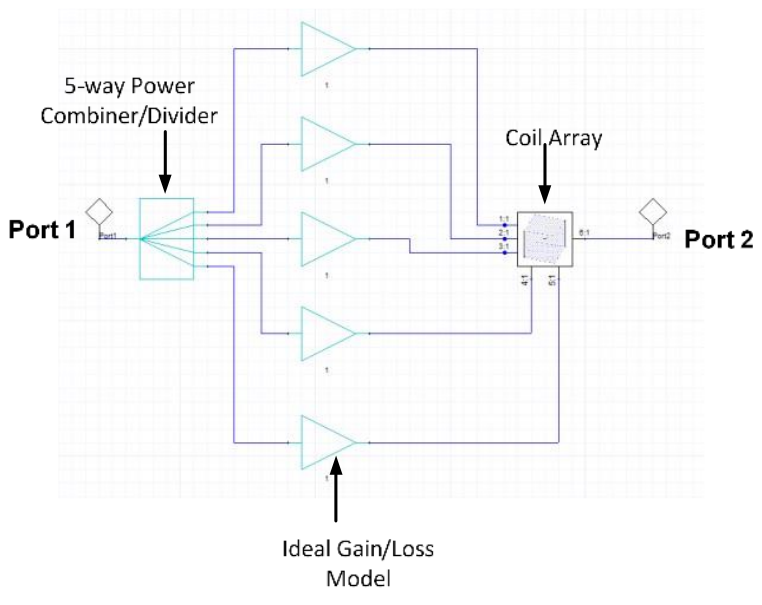

Figure 2. Simulation for the Transmission Coefficient of the coil array

\section{B) Frequency Splitting, Suppression and}

\section{Elimination}

Due to the coupling between adjacent transmitting coils and cross coupling between nonadjacent coils, there is a splitting effect of the received signal at the receiver coil. The behavior of the splitting occurs such that one of the peaks remains at the resonant frequency with the other peak at a higher frequency. The investigation in [5], could not find a way of improving power transfer when there is significant coupling between the transmitter resonant coils.

The splitting effect is suppressed when the coil spacing of the array is increased reducing the coupling between the coils. The splitting effect is eliminated when the coil spacing is such that there is negligible coupling among the transmitter coils as is shown in [5]. However increasing the coil spacing also increases the total area occupied by the coil array which may not feasible in a practical system.

Fig. 3 shows the received signal at the receiver for the 17-turn array as an $S_{21}$ parameter where the transmitter coils are combined as one port and the receiver coil as the other port of a two-port network. Thus the efficiency at the receiver is calculated as in (1) [8].

$$
\text { Efficiency } \eta=\left[S_{21}\right]^{2} \times 100 \%
$$

There is a splitting effect as expected of the received signal which is observed in Fig. 3. There are two peaks at $8.3 \mathrm{MHz}$ and $8.5 \mathrm{MHz}$ respectively. The same phenomenon is observed for the received signal of the 15-turn array with the two peaks at $8.5 \mathrm{MHz}$ and $8.7 \mathrm{MHz}$.

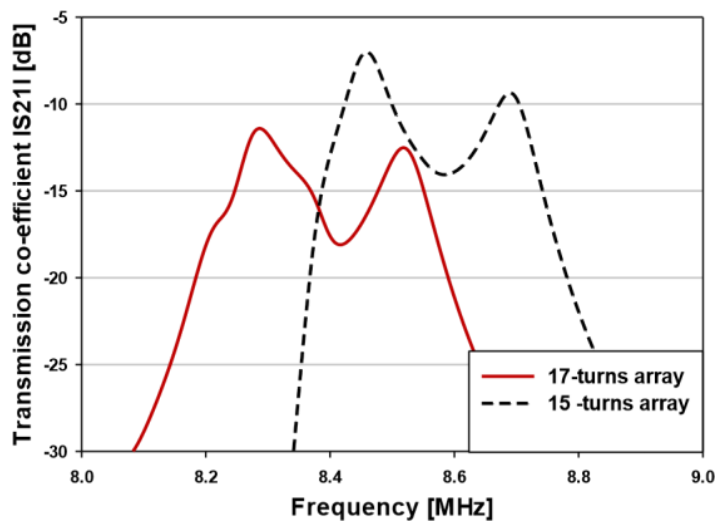

Figure 3. The transmission co-efficient for the coil array

Fig. 4a shows the effect of increasing the coil spacing $s$ between the transmitter coils so as reduce the coupling between the coils for the 17-turns array. The figure shows there is suppression of the splitting effect as the coil spacing is increased until a point where it is eliminated. It is shown that increasing the array spacing from the original distance of $35 \mathrm{~cm}$ to $40 \mathrm{~cm}$ reduces the splitting effect substantially improving the $S_{21}$ value from less than $-10 \mathrm{~dB}$ to just less than $-5 \mathrm{~dB}$. Thus more power reaches the receiver coil. Increasing the distance further improves the received signal with some degree of splitting suppression, however, there is shift in the resonant frequency to a higher frequency. It can be observed that at a spacing distance of $60 \mathrm{~cm}$, there is an $S_{21}$ value of approximately $-5 \mathrm{~dB}$, however, the resonant frequency is around $9 \mathrm{MHz}$. Hence there has to be retuning of the resonant frequency using a directional coupler.

The effect of coil array spacing on the splitting effect suppression for the 15 turn array is shown in Fig. 4b. The same phenomenon of splitting suppression for increasing array spacing is observed. However, the best spacing for this array 
is obtained at $45 \mathrm{~cm}$ and $50 \mathrm{~cm}$. The maximum received signal is observed in this array in the middle of the two resonant frequencies for the spacing of $35 \mathrm{~cm} . \mathrm{S}_{21}$ value of around $-4 \mathrm{~dB}$ is obtained at $8.6 \mathrm{MHz}$ for $50 \mathrm{~cm}$.

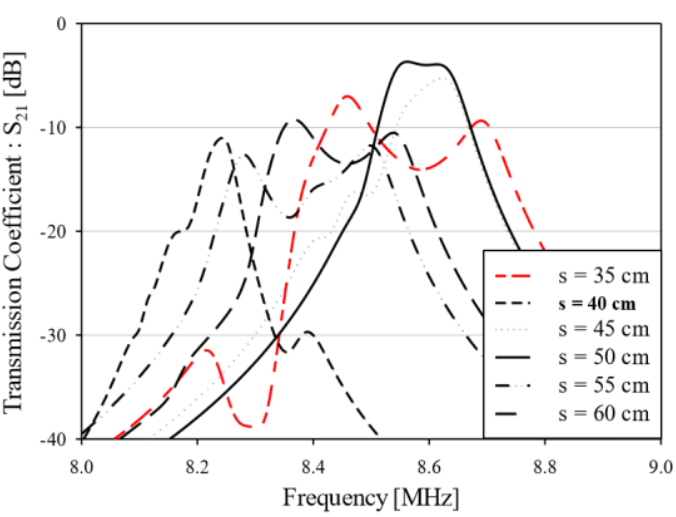

(b)

Figure 4. Effect of the coil array spacing s, on splitting effect (a) 17-turn array (b) 15-turn array

\section{RESULTS AND ANALYSIS}

\section{A) Efficiency Enhancement using horizontal displacement}

Another technique that can also be used to suppress the splitting effect of the coil array is by keeping the coil spacing constant, and displacing horizontally the middle (the second and fourth) coils as is shown in Fig. 5. The change in the transmission co-efficient $\left(\mathrm{S}_{21}\right)$ is observed with the increment of the displacement distance to verify splitting effect suppression.

As the horizontal distance of the coils increases, the splitting is suppressed until it is eventually eliminated as is shown in Fig. 6a. The figure shows the effect of the technique on the 17-turn array with a coil array spacing of $40 \mathrm{~cm}$. It is observed that at a horizontal displacement $h d$ of $10 \mathrm{~cm}$, the resonant frequency shift from the two peaks at 8.3 $\mathrm{MHz}$ and $8.5 \mathrm{MHz}$ to 8.85 and $9.05 \mathrm{MHz}$ respectively. The $S_{21}$ of the structure with $h d=10$ $\mathrm{cm}$ shows the resonance at the first peak (i.e. 8.85 $\mathrm{MHz}$ ), being more dominant. As $h d$ is increased to $20 \mathrm{~cm}$, it is observed that the two resonant peaks begin to combine into one peak at around 8.5 MHz. There is however a small resonant peak at about $8.4 \mathrm{MHz}$. At $h d$ value of $25 \mathrm{~cm}$, the two resonant peaks combine into a single resonant peak at 8.75
MHz. Thus at this distance, the splitting effect is completely eliminated with corresponding increase in the transmission co-efficient value as can be observed. As the $h d$ value is increased further to $30 \mathrm{~cm}$ and over, the splitting effect begins to return with a

substantial decrease in the transmission coefficient as is shown in the figure.

The effect of the horizontal displacement technique as applied to the 15-turns coil array is shown in Fig. 6b. Similarly, the coil array spacing $(s=40 \mathrm{~cm})$ is kept constant while $h d$ is increased. With no horizontal displacement, it is observed that there are two resonant peaks at $8.2 \mathrm{MHz}$ and 8.4 MHz. The resonant peak at $8.2 \mathrm{MHz}$ is however more dominant. Similar resonant peaks are observed for when $h d=20 \mathrm{~cm}$ with slightly lower transmission co-efficient values. The second resonant peak is at $8.55 \mathrm{MHz}$. Two similar resonant peaks are observed for $h d=10 \mathrm{~cm}$ with higher transmission co-efficient (higher than $h d=$ $0 \mathrm{~cm}, 20 \mathrm{~cm})$. The two resonant peaks combine into a single peak for $h d=30 \mathrm{~cm}$. There is also an increase in the transmission coefficient correspondently. Thus the splitting effect is completely eliminated at this distance. The 
splitting effect begins to form again as $h d$ is

increased above $30 \mathrm{~cm}$ as is shown in the figure.

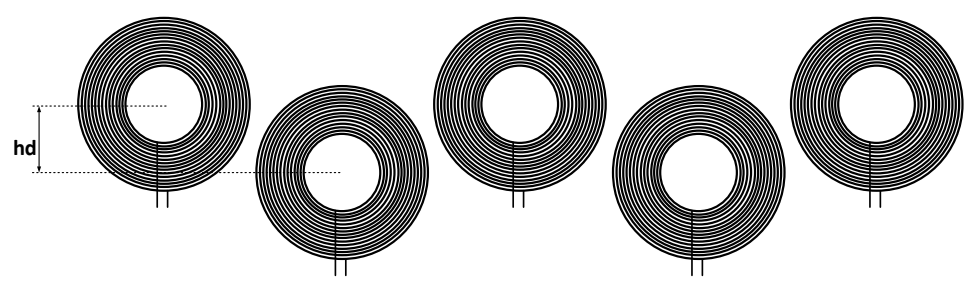

Figure 5. Transmitter coil array showing horizontal displacement of some coils

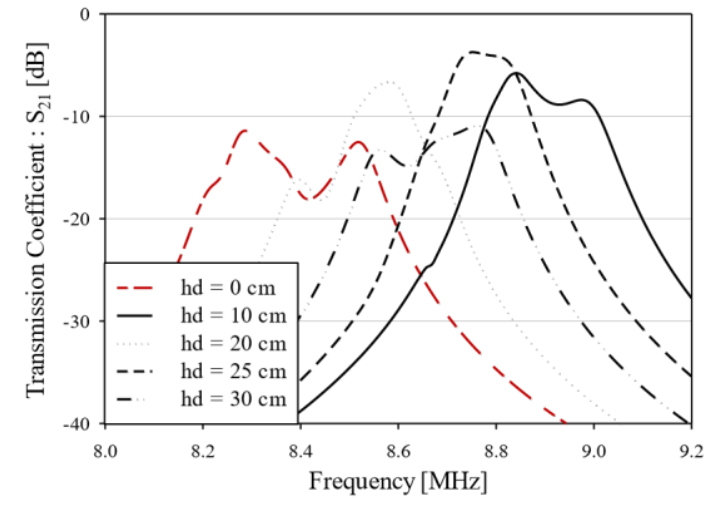

(a)

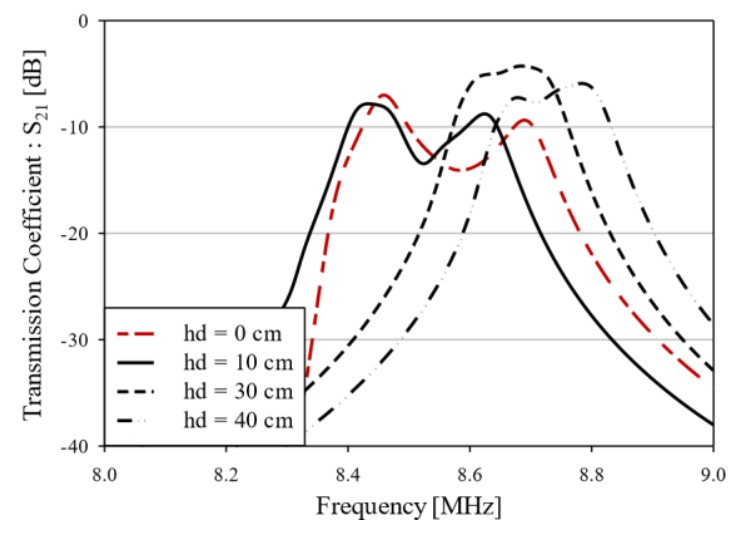

(b)

Figure 6. Effect of horizontal displacement on splitting effect (a) 17-turn array (b) 15-turn array

\section{B) Efficiency Enhancement using Mixed Coil}

Turn-number

To minimize the effect of coupling between the transmitters of the coil array, coils with different turns-number are used in the array design. This design approach helps in the splitting effect suppression and hence produces an improved received signal.

By the use of mutual inductance, frequency splitting and suppression is investigated in [9]. Wireless power transfer via magnetic resonant coupling (WPT/MRC) systems usually have single turn resonant coils as the receiver and transmitter coils with radii of $r_{1}$ and $r_{2}$ respectively. The coils have a transfer distance of $d$ as the coils are placed face-to-face. The mutual inductance between these coils is found by the function $M(d)$ which is defined as (2) [9]

$$
\left\{\begin{array}{c}
M(d)=\mu_{0} \frac{\sqrt{r_{1} r_{2}}}{g}\left[\left(2-g^{2}\right) K\left(g^{2}\right)-2 E\left(g^{2}\right)\right] \\
g^{2}=\frac{4 r_{1} r_{2}}{d^{2}+\left(r_{1}+r_{2}\right)^{2}}
\end{array}\right.
$$

(2)

where $\mathrm{K}(*)$ and $\mathrm{E}(*)$ are respectively the complete elliptic integrals of the first and second kind [9]. Generally, WPT systems use similar dimensions for the transmitting and receiving coils. For the case where $\left(r_{1}=r_{2}\right), g$ approaches 1 rapidly as the receiving coil moves close to the transmitting coil (i.e when $d \rightarrow 0$ ) [9]. The optimal transfer consideration is destroyed and frequency splitting occurs because $K(1)=\infty$ and $E(1)=1$. Thus $M(d)$ increases rapidly while there is a decrease of the $d$ between the two coils [9].

Frequency splitting is eliminated by the maintenance of $g$ much less than 1 for all distances to avoid the pole $K(1)=\infty$. This is achieved by having unequal $r_{1}$ and $r_{2}$ which causes the pole of 
$M(d)$ to disappear [9]. Applying the concept of non-identical coils with different transmitting and receiving coil radii as discussed above to the multiple transmitter coil array, helps to suppress the splitting effect due to the uniform coupling among the transmitters with tight coupling.

The design approach is to change the number of turns used for the individual coils for the coils that are in between transmitters. Therefore for a fivecoil array, the second and fourth coils are the ones of interest. The 17-turns coil array is used as the base array for this design approach. The second and fourth coils are replaced with 15-turns single coils to form a new mixed number of turns coil array. Thus a multiple coil array is formed with 17turns single coils at the first, third and fifth positions and 15-turns single coils at the second and fourth positions. This mixed number of turns coil array is the first of three types of such arrays designed. The coil array with mixed 17turns and 15-turns coils is shown in Fig. 7(a).

The second mixed array is made with a 13-turns single coil at the second and fourth positions while the third array is designed with an 11-turns single coil. These structures are shown in Fig. 7(b) and Fig. 7(c) respectively.

The transmitter coils are set to be very close to each other, hence $s$ is set to be $35 \mathrm{~cm}$ for all the three designs. The effect of this design approach is verified by observing the transmission coefficient of the array. The $S_{21}$ values of the mixed-turns array are shown in Fig. 8. It is observed that with the mixed 17-turns and 15-turns array, the two resonant peaks that are obtained with the 17-turns and 15-turns array are closer. This increases the $S_{21}$ value for this mixed coil array which is higher than the value for the all 17-turns and 15-turns structures. The resonant peaks are at $8.4 \mathrm{MHz}$ and 8.55 MHz respectively. The resonant peaks are combined for the 17-turns and 13-turns array structure. However, the resonant frequency is shifted to $9.15 \mathrm{MHz}$ and requires retuning. The same phenomenon is observed for the 17-turns and 11-turns array with the single resonant peak at 8.8 $\mathrm{MHz}$.

It has been verified that to reduce the coupling between the transmitter coils in a coil array, which causes a splitting effect, mixed coil turns can be used.

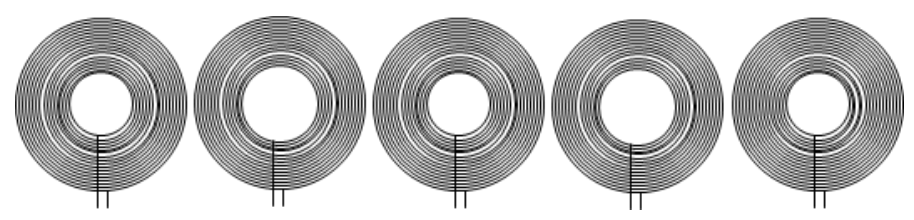

17- turns 15- turns 17- turns 15- turns 17 - turns

(a)

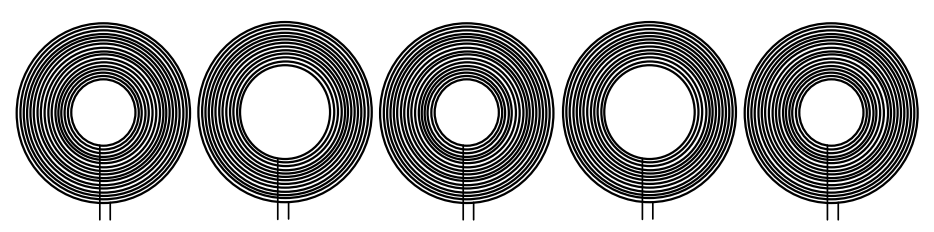

17- turns 13- turns 17- turns 13-turns 17- turns

(b)

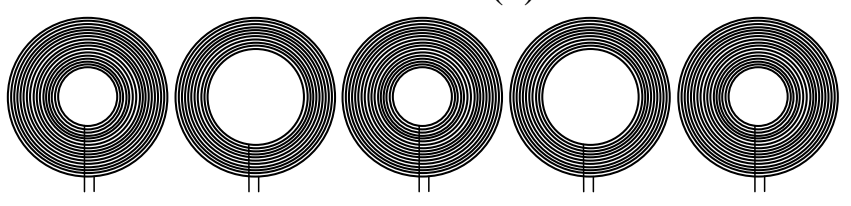

17- turns 11- turns 17- turns 11- turns 17- turns

(c) 
Figure 7. Mixed turns coil array (a) 17 turns and 15 turns (b) 17 turns and 13 turns (c) 17 turns and 11 turns

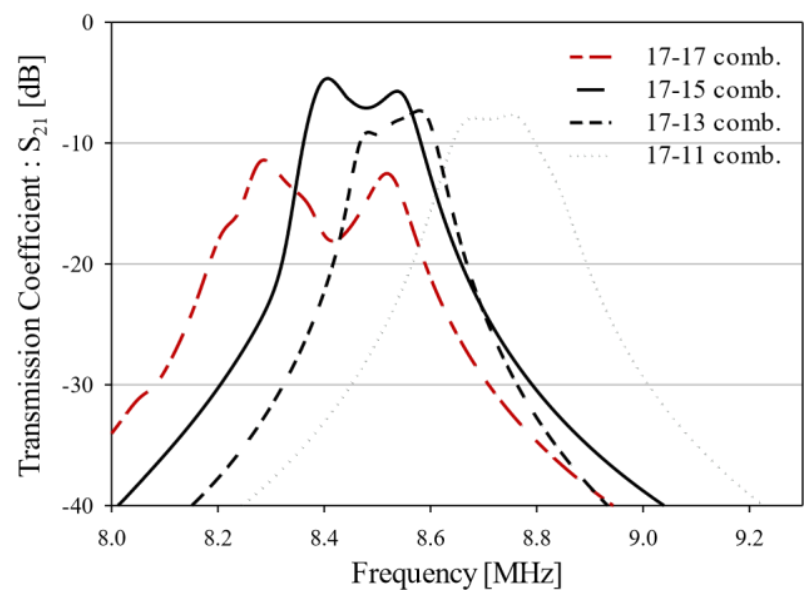

Figure 8. Effect of mixed turns coil array on splitting effect

\section{C) Efficiency Enhancement using Stacked Coils}

To increase the received signal, stacked coils are placed at distances from the transmitter coil array. The magnetic power is coupled from the array to the stacked coils in order for higher signal power to reach the receiver coil.

The base coil array used to verify the effect of the use of stacked coils is the 17-turns and 15turns mixed coil array. The stacked coils are made up of the same coil array without the input voltage and the series capacitors. First the distance between the coil array and the stacked coils $(s h)$ is considered to find the optimum distance for the stacked coils as shown in Fig. 9a. Then at a particular sh value the stacked coils are displaced horizontally as illustrated in Fig. 9b.

The distance from the stacked coils from the coil array has a big impact on the received signal. The closer the stacked coils are to the coil array, the lower the received signal. As can be seen in Fig. 10a, $s h$ value of $2 \mathrm{~cm}$ produce an $\mathrm{S}_{21}$ value of $-25 \mathrm{~dB}$. The $\mathrm{S}_{21}$ increases as $s h$ increases with 20 $\mathrm{dB}$ and $-12 \mathrm{~dB}$ for $s h$ of $5 \mathrm{~cm}$ and $8 \mathrm{~cm}$ respectively. However, as $s h$ is increased past $8 \mathrm{~cm}$ the received signal experiences splitting effect and thereby reducing the $S_{21}$.

The stacked coils displacement $s d$, increases the received signal significantly. As is shown in Fig. $10 \mathrm{~b}$, the higher the displacement the higher the received signal. The height of the stacked coils is set at $5 \mathrm{~cm}$ from the coil array. It is observed that, with $s d=5 \mathrm{~cm}$, the $S_{21}$ is $-16.7 \mathrm{~dB}$. The $S_{21}$ increases sharply to $-5.8 \mathrm{~dB}$ for $s d=10 \mathrm{~cm}$. $\mathrm{S}_{21}$ value of $-2.9 \mathrm{~dB}$ is obtained with $\mathrm{sd}=15 \mathrm{~cm}$. Further increase in $s d$ causes a splitting effect with the resonant peaks at $8.8 \mathrm{MHz}$ and $8.9 \mathrm{MHz}$ having $\mathrm{S}_{21}$ values of $-3.9 \mathrm{~dB}$ and $-4.9 \mathrm{~dB}$ respectively. Thus the optimum displacement of the stacked coils is $s d=15 \mathrm{~cm}$. 


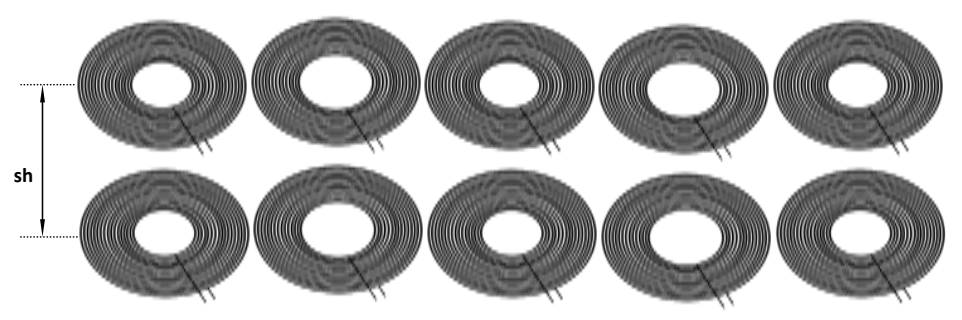

(a)

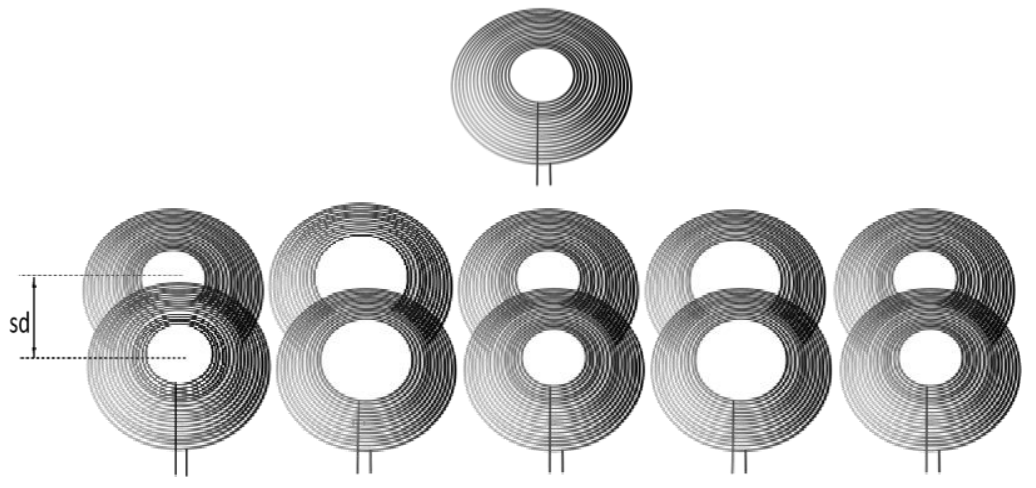

(b)

Figure 9. Stacked coil array structure (a) Mixed 17-turns and 15-turns coils with stacked coils (b)

Displacement of the stacked coils

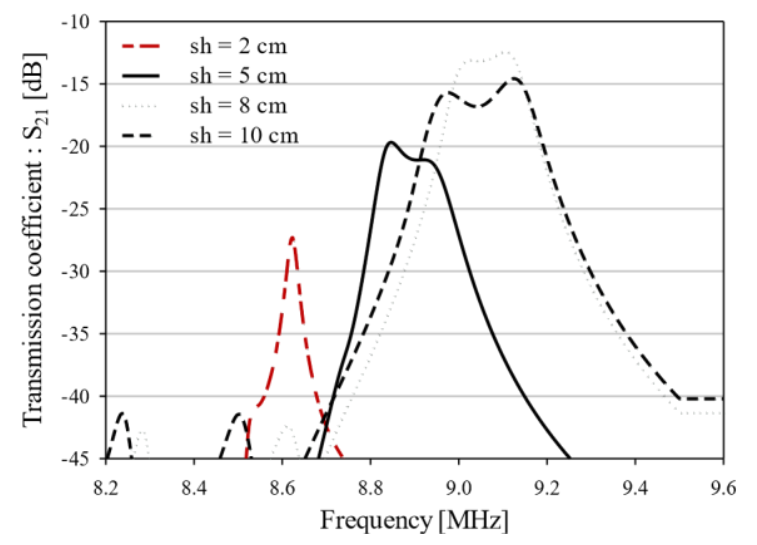

(a)

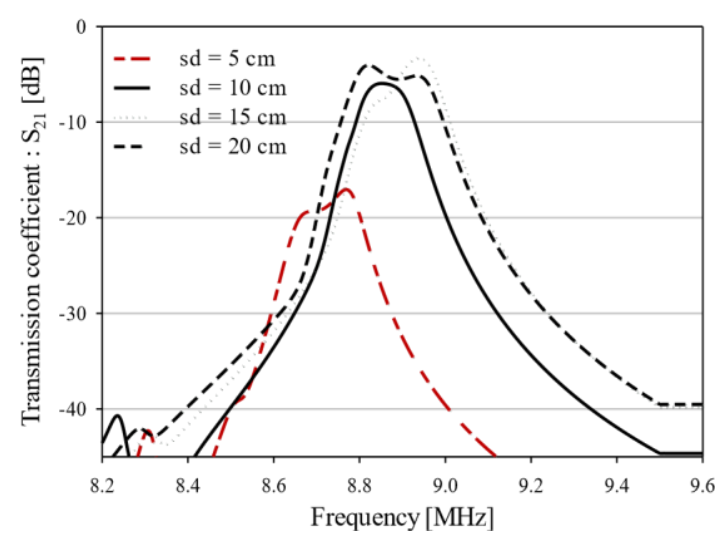

(b)

Figure 10. Effect of stacked coils on efficiency (a) Effect of the height of the stacked coils from the coil array (b) Effect of the displacement of the stacked coils

\section{D) Array port position on transfer Efficiency}

The position and orientation of the port of the transmitter coil in relation to the receiver coil has an impact on the transfer efficiency. To investigate this phenomenon, the transfer efficiency of a single transmitter to a single receiver is first analyzed. The port position of the receiver is fixed while the port position of the transmitter is varied as is illustrated in Fig. 11. The effect of the transmitter port positions on the efficiency is analyzed both for the 17-turns and 15-turns coils. It is observed that for the 17-turns coils, the transfer efficiency tendency are similar for port positions 1 and 2 with position 1 having better efficiency performance than position 2 as is shown in Fig. 12(a). The efficiency of position 3 is similar to that of position 1 but at a slightly higher frequency. The lowest transfer efficiency performance is observed for port position 4 . 
Thus the port position of the transmitter in relation to the receiver coil determines the absolute value and the sign of the mutual inductance between the transmitter coil and the receiver coil. The port position also determines the current direction and hence the direction of the magnetic field generated by the transmitter resulting in increased transfer power to the receiver coil

For the 15-turns, port position 4 has the lowest transfer efficiency just as is the case for the 17turns coil as is seen in Fig. 12(b). Port positions 2 and 3 have similar efficiencies with position 2 having a slightly better performance. Port position 1 has the highest efficiency performance

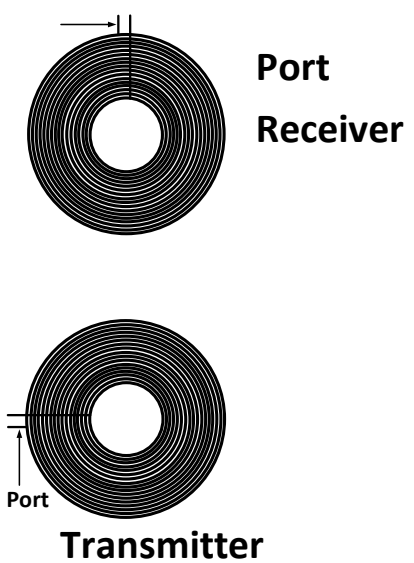

(a)
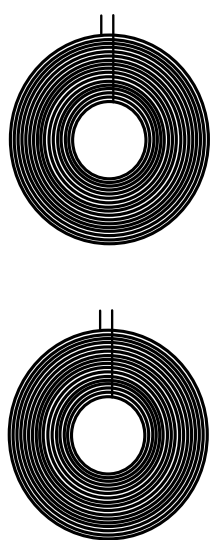

(b)
To verify the transmitter port positions in the coil array, the four port positions are applied to the final transmitter array structure (mixed coil turns with displaced stacked coils). The transfer efficiencies of the array for the receiver at the boresight are observed. The transmitter array structures showing the different port positions are shown in Figs. 13a to13d. The transmission coefficient plots for the receiver is shown in Fig. 14 with Table 1 showing the comparison of the efficiencies for the different structures. The plots show that the array using the port position 1 achieves the best efficiency performance of $36.9 \%$ with port position 3 achieving the lowest efficiency
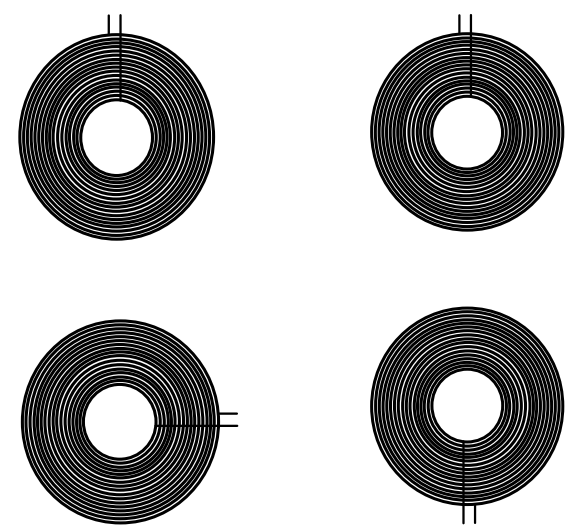

(c)

(d)

Figure 11. Transmitter coil port positions (a) Position 1 (b) Position 2 (c) Position 3(d) Position 4

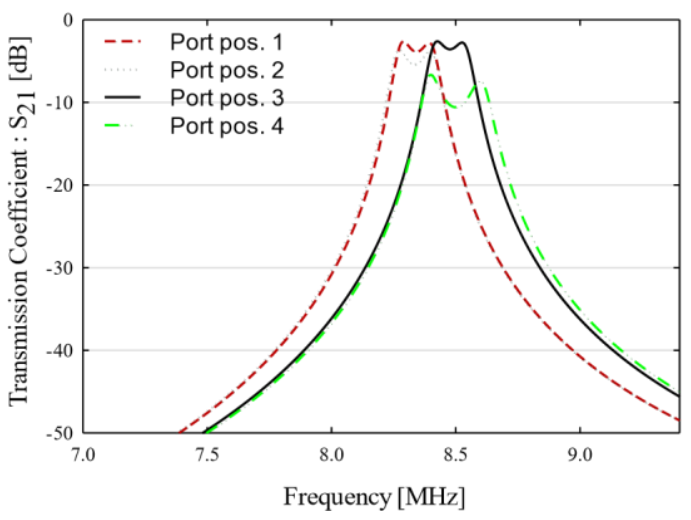

(a)

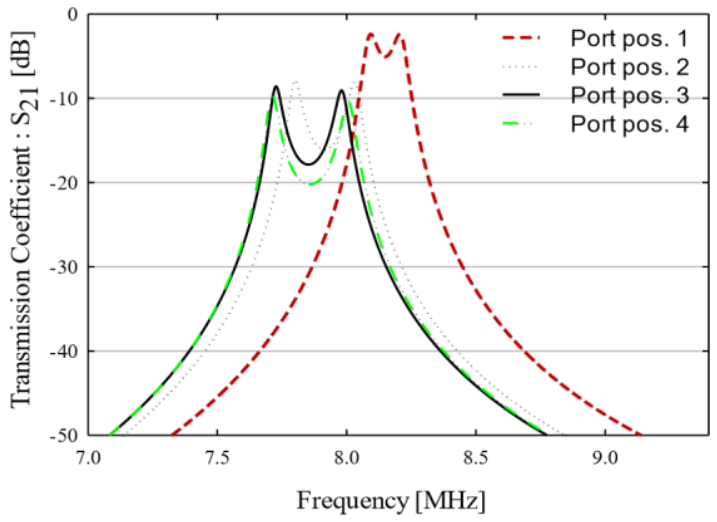

(b)

Figure 12. Transmission Coefficient for port positions (a) 17-turns (b) 15-turns 


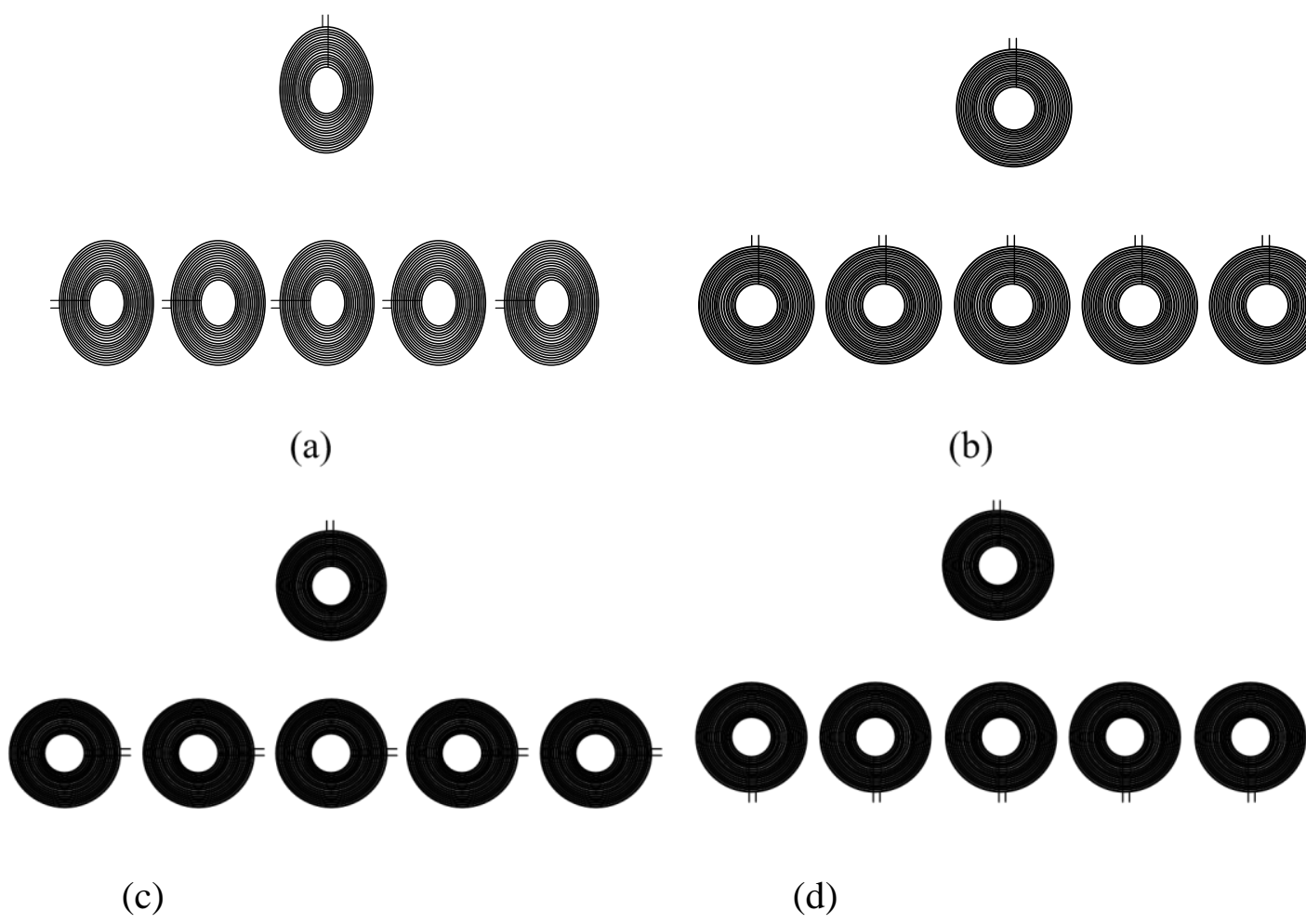

Figure 13. Structure of transmitter coil array using port positions (a) With port position 1 (b) With port position 2 (c) With port position 3 (d) With port position 4

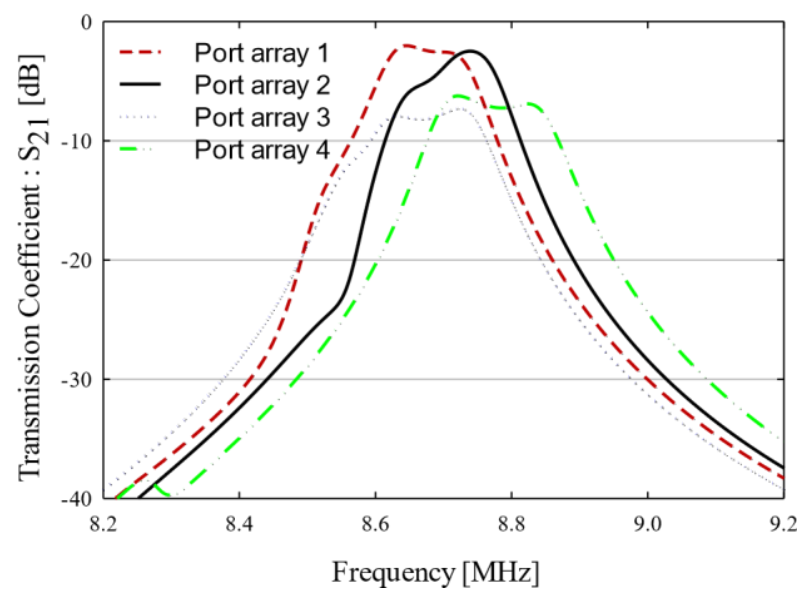

Figure 14. Transmission coefficient of array using port positions

Table -1 Port positions and efficiency for coil array

\begin{tabular}{|c|c|c|}
\hline Port array positions & Efficiency [\%] & Optimal Frequency [MHz] \\
\hline 1 & 36.9 & 8.6 \\
\hline 2 & 31.8 & 8.7 \\
\hline 3 & 3.4 & 8.7 \\
\hline 4 & 5.6 & 8.7 \\
\hline
\end{tabular}

\section{IV.CONCLUSION}

In this paper, a multiple transmitter coil array with a single receiver coil is designed. The array is designed with a 2.54 copper coil with 17-turns and 15 turns having five transmitter coils. The linear array was first designed with all 17-turns and 15- 
turns transmitters. The frequency splitting effect as a result of the coupling between the resonant transmitter coils due to their close proximity was verified. It was observed that due to the coupling between the transmitters, two resonant peaks are attained one at the resonant frequency and the other at a higher or lower frequency. The effect of the array spacing of the coil array on the suppression of the splitting effect was verified by increasing the distances between the coils and observing the effect on the transmission co-efficient of the combined signal at the receiver coil placed $40 \mathrm{~cm}$ away. It is observed that the splitting effect is suppressed and eliminated by the increase of the array spacing.

The horizontal displacement of the middle coils (second and fourth coils of the 5-coil array) of the transmitter array with constant spacing was shown also to suppress the splitting effect due to coupling. It is shown that the splitting effect was suppressed as the displacement distance is increased. An optimum value of $30 \mathrm{~cm}$ is found to eliminate the frequency splitting effect.

To eliminate the splitting effect with a small total array area, the design of a multiple transmitter array with different coil turns was proposed. It has been shown that designing the array with the middle coils having different number of turns achieves uniform coupling among the coils reducing the splitting effect. It was shown that designing the coil array with 17-turn coils with 15turn coils as the middle coils significantly reduces the splitting effect increasing the transfer efficiency at the receiver coil. Splitting was eliminated when the array was designed having 17turns with 13-turns or 11-turns in between. However there were frequency shifts with these designs requiring retuning. The 17-turns and 15turns mixed array were selected because of their higher efficiencies compared to the other mixed turns arrays.

The introduction of stacked coils on top of the transmitter coil array was shown to improve the efficiency of the received signal. Maximum power reaches the receiver coil when the stacked coils were placed at $5 \mathrm{~cm}$ from the coil array. It is also shown that, the displacement of the stack coil increases the efficiency at the receiver coil with a displacement of $15 \mathrm{~cm}$ achieving the highest efficiency. The displaced stacked coils increases the transfer efficiency from $15.85 \%$ to $26.30 \%$ for the transfer distance of $40 \mathrm{~cm}$.

The position and orientation of the ports of the array in relation to the receiver coil were shown to have an impact on the transfer efficiency. Analysis and optimization of different array port arrangements increased the transfer efficiency to $36.9 \%$

\section{ACKNOWLEDGEMENT}

This work was supported by Institute of Information \& communications Technology Planning \& Evaluation(IITP) grant funded by the Korea government(MSIT) (No. 2017-0-00653, Research on middle- and long-range wireless power transfer core technologies using magnetic field)

\section{REFERENCES}

[1] N. Shinohara, Wireless Power Transfer via Radiowaves, John Wiley \& Sons, 2014.

[2] J. I. Agbinya, Wireless Power Transfer, River Publishers, 2015.

[3] A. P. Sample, D. A. Meyer, and J. R. Smith, "Analysis, experimental results, and range adaptation of magnetically coupled resonators for wireless power transfer," IEEE Trans. Ind. Electron., vol. 58, no. 2, pp. 544-554, Feb. 2011.

[4] C. Zhang, D. Lin and S. Y. R. Hu, "Efficiency optimization method of inductive coupling wireless power transfer system with multiple transmitters and single receiver," in Energy Conversion Congress and Exposition (ECCE), Milwaukee, WI, USA, 2016.

[5] R. Johari, J. V. Krogmeier and D. J. Love, "Analysis and Practical Considerations in Implementing Multiple Transmitters for Wireless Power Transfer via Coupled Magnetic Resonance," IEEE Transactions on 
Industrial Electronics, vol. 61, no. 4, pp. 774 1783, May 2013.

[6] J. Jadidian and D. Katabi, "Magnetic MIMO: How to charge your phone in your pocket," in Proc. Annu. Int. Conf. Mobile Comput. Netw. (MobiCom'14), Sep. 2014.

[7] W. Q. Niu, J. X. Chu, W. Gu, and A. D. Shen, "Exact analysis of frequency splitting phenomena of contactless power transfer systems," IEEE Trans. Circuits Syst. I, Reg. Papers, vol. 60, no. 6, pp. 1670-1677, Jun. 2013.

[8] V. T. Nguyen, S. H. Kang, J. H. Choi and C. W. Jung, "Magnetic resonance wireless power transfer using three-coil system with single planar receiver for laptop applications," IEEE Transactions on Consumer Electronics, vol. 61, no. 2, pp. 160-166, May 2015.

[9] Y.-L. Lyu, F.-Y. Meng and et.al, "A Method of Using Nonidentical Resonant Coils for Frequency Splitting Elimination in Wireless Power Transfer," IEEE Transactions on Power Electronics, vol. 30, no. 11, pp. 6097-6107, Nov. 2015. 